\title{
Gender Differences in Electrophysiological Characteristics of Idiopathic Ventricular Tachycardia Originating From Right Ventricular Outflow Tract
}

\author{
S.-G. YANG ${ }^{1,2}$, M. MLČEK ${ }^{2}$, O. KITTNAR ${ }^{2}$ \\ ${ }^{1}$ Institution of Cardiology, Taipei Hospital in Taiwan, Republic of China, ${ }^{2}$ Institute of Physiology, \\ First Faculty of Medicine, Charles University in Prague, Czech Republic
}

Received March 11, 2014

Accepted May 16, 2014

\section{Summary}

It has become increasingly apparent in recent years that there are important differences of many cardiovascular disorders including ventricular tachycardias in men and women. Nevertheless, so far just few studies have addressed possible gender differences in electrophysiological characteristics of idiopathic ventricular tachycardia from right ventricular outflow tract (RVOT-VT), other than epidemiological ones. This study explored possible gender differences in electrophysiological characteristics and catheter ablation outcome in RVOT-VT patients. Ninety-three patients (mean age $38.7 \pm 15.5$ years, 30 males) with idiopathic RVOT-VT were enrolled and analyzed in our study. Male patients had longer QRS width ( $99.9 \pm 19.4 \mathrm{~ms}$ vs. $88.4 \pm 20.7 \mathrm{~ms}, \mathrm{p}=0.02)$. Female patients had lower right ventricular mean voltage $(3.0 \pm 0.7 \mathrm{mV}$ vs. $3.7 \pm 0.9 \mathrm{mV}, \mathrm{p}=0.03$ ), and more low voltage zone over the right ventricular outflow tract free wall $(27.0 \%$ vs. $6.7 \%, p=0.02)$. Eighty-one patients passed catheter ablation ( 23 males). The acute success rate, repeated catheter ablation rate and VT recurrence rate were similar in both genders. The present study provides evidence of the gender differences in electrophysiological findings in patients with idiopathic RVOT-VT. Studies on gender-specific differences in arrhythmia could lead to a better understanding of its mechanism(s) and provide valuable information for the development of optimal treatment strategies.

\section{Key words}

Gender difference - Electrophysiological characteristics • Idiopathic ventricular

\section{Corresponding author}

O. Kittnar, Institute of Physiology, Charles University in Prague, First Faculty of Medicine, Albertov 5, 12800 Prague 2, Czech Republic. E-mail: okittnar@lf1.cuni.cz

\section{Introduction}

Idiopathic ventricular tachycardia (IVT) is a special type of monomorphic ventricular tachycardia without any morphological cardiac abnormality. Its management and prognosis differ from ventricular tachycardia associated with structural heart disease and it represents approximately $10 \%$ of all ventricular tachycardias in adult patients. According to the long-term experience they have relatively very good prognosis. IVTs are usually classified into three groups according to the site of their origin: IVT from right ventricular outflow tract (RVOT-VT), IVT from left ventricular outflow tract and Fascicular IVT. RVOT-VT is a common arrhythmia in young patients without any structural heart disease. The arrhythmia is characterized by repetitive bursts and premature ventricular beats with electrocardiographic patterns of a left bundle branch block, inferior-axis QRS morphology, and symptoms of palpitations (Marchlinski et al. 2000).

It has become increasingly apparent in recent years that there are important differences of many cardiovascular disorders including ventricular tachycardias in men and women. Gender differences have been observed in the epidemiology, pathogenesis and clinical presentation of various ventricular arrhythmias (Yarnoz and Curtis 2008, Nakagawa et al. 2002). For example, Brugada's syndrome is more common in men than in women, while idiopathic RVOT-VT, drug-related Torsade de Pointes and long QT syndrome are more common in women. Significantly higher mortality rate and sudden death rate have been documented in male patients with 
arrhythmogenic right ventricular cardiomyopathy/dysplasia (ARVC/D) than in female patients with the same mutation (Hodgkinson et al. 2005). Females are also more susceptible to drug-induced torsade de pointes (TdP) and in one recent study they were proved to have greater QTc prolongation than males following sotalol administration (Somberg et al. 2012) what can explain the higher incidence of drug-induced TdP seen in females.

Studies have identified triggers of RVOT-VT and found women tended to have frequent VT initiation during hormonal flux (Marchlinski et al. 2000). Nevertheless, so far just few studies have addressed possible gender differences in electrophysiological characteristics of idiopathic RVOT-VT, other than epidemiological ones. Regarding the gender differences in electrocardiographic findings the large study of Huang and co-workers suggested that clinical and electrophysiological characteristics of patients with accessory atrioventricular pathways could be closely linked and may imply a different pathogenesis (Huang et al. 2011). However it could be generally concluded that the identifiable electro-anatomical characteristics of each gender are still not yet understood clearly. Therefore we have designed this study to contribute to understanding of this question by exploring gender differences in electrophysiological characteristics and the results of catheter ablation in patients suffering from idiopathic RVOT-VT.

\section{Methods and Patients}

\section{Study population}

93 patients with idiopathic RVOT-VT (mean age $38.7 \pm 15.5$ years) were enrolled into the study between
January 2011 and September 2013. As shown in Table 1, there was no difference between genders in age of disease onset, diabetes mellitus, hyperlipidemia, previous syncope episodes or family history of ventricular arrhythmias. The diagnosis of VT was documented either by 12-lead resting electrocardiogram (ECG) or by 24-hour ambulatory ECG according to Holter. Cardiac catheterization excluded the possibility of coronary artery disease. All patients were examined using transthoracic echocardiography at the time of diagnosis and all other heart diseases, such as dilated cardiomyopathy, were excluded. Right ventricular (RV) function, regional RV wall motion, as well as left ventricular (LV) function, LV wall motion and LV ejection fraction (EF) were evaluated. RV dysfunction was defined as regional RV akinesia, RV dyskinesia, RV aneurysm, or RV EF less than $40 \%$. Arrhythmogenic right ventricular cardiomyopathy/dysplasia (ARVC/D) was excluded by Task-Force criteria (Marcus et al. 2010). Monomorphic VT was defined as VT with a uniform beat-to-beat surface QRS morphology. Electrocardiographic criteria were employed to identify VT origin, and in all patients included into the study the VTs were confirmed to be of RVOT-VT type (Arya et al. 2007).

Electrophysiological study and electromechanical mapping

Standard electrophysiological study was performed on all patients after discontinuation of antiarrhythmic agents for more than five half-lives. Programmed ventricular electrical stimulation (Hummel et al. 1994) was performed with up to three extrastimuli delivered during sinus rhythm after eight paced ventricular cycle lengths. Induced VT with duration

Table 1. Baseline characteristics of male and female patients with idiopathic RVOT-VT.

\begin{tabular}{lccc}
\hline & Males $(\mathbf{n}=\mathbf{3 0})$ & Females $(\mathbf{n}=\mathbf{6 3})$ & p value \\
\hline Age onset (years) & $44.8 \pm 18.3$ & $36.7 \pm 14.1$ & NS \\
BMI $\left(\mathrm{kg} / \mathrm{m}^{2}\right)$ & $23.8 \pm 2.7$ & $24.0 \pm 6.8$ & NS \\
Syncope & $43.3 \%$ & $34.9 \%$ & NS \\
Hypertension & $20.0 \%$ & $17.5 \%$ & $\mathrm{NS}$ \\
Diabetes mellitus & $10.0 \%$ & $7.9 \%$ & $\mathrm{NS}$ \\
Hyperlipidemia & $20.0 \%$ & $6.3 \%$ & $\mathrm{NS}$ \\
Family history of ventricular & $3.3 \%$ & $3.2 \%$ & NS \\
arrhythmias & & & \\
\hline
\end{tabular}

BMI, body mass index; RVOT-VT, right ventricular outflow tract-ventricular tachycardia 
longer than $30 \mathrm{~s}$ or concomitant hemodynamic compromise was classified as inducible sustained VT. The first test location was at the RV apex, while the next test site was the RVOT if sustained VT was not induced from the previous site. When electrical stimulation failed to induce sustained VT, we used intravenous infusion of isoprenaline (1-4 $\mu \mathrm{g} / \mathrm{min})$ with or without atropine. Sustained VT induced under the use of isoprenaline with or without atropine was defined as catecholaminesensitive VT. We analyzed the location of the RVOT-VT origin using multiplane fluoroscopy, mainly in the $60^{\circ}$ left anterior oblique projection and the $30^{\circ}$ right anterior oblique projection. If the VT origin was located to the anterior or posterolateral attachment of the RVOT the patient was thought to have VT of a septal origin (Tada et al. 2004).

We performed simultaneous electromechanical mapping during electrophysiological study (EPS) using the NavX mapping system (NavX, St. Jude Medical, St. Paul, $\mathrm{MN}$, USA). The mapping procedure included pace mapping during sinus rhythm, endocardial activation mapping, identification of diastolic potentials and entrainment mapping during VT. Entrainment was performed to identify the critical component of the VT circuit and for guidance of selective ablation. We used endocardial activation-sequence mapping to record the earliest endocardial activity and diastolic potentials during VT. Continuous recordings of RV endocardium voltage were done during sinus rhythm, and abnormal areas were defined as voltage setting of $\leq 1.5 \mathrm{mV}$ on bipolar electrocardiogram. Scar zones were defined by a voltage setting $<0.5 \mathrm{mV}$, whereas areas with voltage between $0.5 \mathrm{mV}$ and $1.5 \mathrm{mV}$ were evaluated as low voltage zones. This facilitates the delineation of the culprit substrate for VT. We performed catheter ablation in those patients with inducible VT with standard radiofrequency energy delivered through 4-mm tipped deflectable ablation catheters. Twelve patients did not receive catheter ablation because relevant clinical VT was not induced during EPS. Acute success was defined by the absence of any inducible VT at the end of the catheter ablation procedure via electrical stimulation with or without isoprenaline. ECGs were checked soon after EPS or catheter ablation.

After hospital discharge, the first outpatient follow-up time was arranged two weeks later and further follow-up visits were scheduled at three-month intervals. Surface ECGs and 24-hour Holter ECG exams were arranged during serial outpatient follow-up. VT with RVOT origins documented on ECGs or 24-hour Holter ECG exams was classified as VT recurrence.
All measurements were performed in the morning between 8 a.m. and 12 a.m. In our study we have used only standard measurement procedures, more detailed description of methods is available in cited papers (Hummel et al. 1994, Tada et al. 2004).

\section{Statistical analysis}

Continuous variables are expressed as mean \pm standard deviation and the comparisons between continuous data were performed using Student's t test. Comparisons of categorical data were performed using a Chi-square test with a Yates' correction or Fisher's exact test. Statistical significance was established at a $p$ value of $<0.05$. All statistical analyses were performed using commercial statistical SPSS version 17.0 software (SPSS, Chicago, IL, USA).

\section{Results}

Electrocardiographic and echocardiographic differences

No differences existed between genders in respect to percentage of atrial arrhythmias and QTc prolongation. We found an RBBB pattern of QRS in four male patients $(13.3 \%)$ and five female patients $(7.9 \%)$, which was not statistically significant. The QRS width was longer in men comparing to women $(99.9 \pm 19.4 \mathrm{~ms}$ vs. $88.4 \pm 20.7 \mathrm{~ms}, \mathrm{p}=0.02)$. The incidence of $\mathrm{T}$-wave inversion in right precordial leads $\left(\mathrm{V}_{1}\right.$ to $\left.\mathrm{V}_{3}\right)$ indicated no statistical differences between genders. Twenty male patients $(66.7 \%)$ presented with clinically documented VT, while 50 female patients $(79.4 \%$ ) did. Most of them had monomorphic VT. Only two male patients and three female patients had multiple monomorphic VT or polymorphic VT (Table 2).

There was no difference in LVEF between both genders. Twenty-one percent of male patients had RV dysfunction compared to $19 \%$ of female patients $(\mathrm{p}=0.85)$. The percentage of RV dyskinesia was similar for male and female patients $(13.8 \%$ vs. $20.6 \%, \mathrm{p}=0.43)$.

\section{Electrophysiology study and electro-anatomic mapping}

Male patients were found to have higher mean $\mathrm{RV}$ voltage than female patients $(3.7 \pm 0.9 \mathrm{mV}$ vs. $3.0 \pm 0.7$ $\mathrm{mV}, \mathrm{p}=0.03)$. Analyzing scar zones and low voltage zones at RVOT and RV, females had more low voltage zones at the RVOT free wall ( $27 \%$ vs. $6.7 \%, \mathrm{p}=0.02)$ as compared with males. The percentage of low voltage zones in other areas was similar between both genders (male vs. female, RVOT septum, $10 \%$ vs. $12.7 \%$, $\mathrm{p}=0.50$; RV body free wall, $16.7 \%$ vs. $30.2 \%, \mathrm{p}=0.17$; 
Table 2. Electrocardiography and echocardiography characteristics in male and female patients with idiopathic RVOT-VT.

\begin{tabular}{|c|c|c|c|}
\hline & Males $(\mathbf{n}=\mathbf{3 0})$ & Females $(n=63)$ & p value \\
\hline Atrial arrhythmia & $30.3 \%$ & $14.3 \%$ & NS \\
\hline$Q T c(m s)$ & $418.6 \pm 34.4$ & $432.3 \pm 41.2$ & NS \\
\hline QTc prolonged $(\geq 440 \mathrm{~ms})$ & $29.6 \%$ & $34.5 \%$ & NS \\
\hline$R B B B$ & $13.3 \%$ & $7.9 \%$ & NS \\
\hline QRS width (ms) & $99.9 \pm 19.4$ & $88.4 \pm 20.7$ & 0.02 \\
\hline Repolarization abnormalities* & $10.0 \%$ & $19.0 \%$ & NS \\
\hline Clinically documented VT & $66.7 \%$ & $79.4 \%$ & NS \\
\hline$L V E F$ & $0.58 \pm 0.8$ & $0.57 \pm 0.8$ & NS \\
\hline$L V$ enlargement & $3.4 \%$ & $1.6 \%$ & NS \\
\hline LV hypokinesis & $3.4 \%$ & $6.3 \%$ & NS \\
\hline$R V$ enlargement & $6.9 \%$ & $6.3 \%$ & NS \\
\hline$R V$ regional dyskinesia & $13.8 \%$ & $20.6 \%$ & NS \\
\hline
\end{tabular}

$\mathrm{EF}$, ejection fraction; LV, left ventricle; QTc, QT interval corrected for heart rate; RBBB, right bundle branch block; RV, right ventricle; RVOT-VT, right ventricular outflow tract-ventricular tachycardia; VT, ventricular tachycardia. * T wave inversion in right precordial leads $\mathrm{V}_{1}-\mathrm{V}_{3}$

Table 3. Electroanatomical mapping characteristics for male and female patients with idiopathic RVOT-VT.

\begin{tabular}{|c|c|c|c|c|}
\hline & & Males $(n=30)$ & Females $(n=63)$ & p value \\
\hline Mean $R V$ voltage & & $3.7 \pm 0.9$ & $3.0 \pm 0.7$ & 0.03 \\
\hline $\begin{array}{l}\text { Low voltage zones } \\
\text { wall }\end{array}$ & RVOT free & $6.7 \%$ & $27.0 \%$ & 0.02 \\
\hline Low voltage zones & RVOT septum & $10.0 \%$ & $12.7 \%$ & NS \\
\hline Low voltage zones & $R V$ free wall & $16.7 \%$ & $30.2 \%$ & NS \\
\hline Low voltage zones & $R V$ septum & $6.7 \%$ & $0.6 \%$ & NS \\
\hline
\end{tabular}

RV, right ventricle; RVOT, right ventricular outflow tract

Table 4. Ablation results for male and female patients with idiopathic RVOT-VT receiving catheter ablation.

\begin{tabular}{lccc}
\hline & Males (n=23) & Females (n=58) & p value \\
\hline HR (beats/minute) & $71.1 \pm 12.2$ & $72.0 \pm 15.1$ & NS \\
Inducible sustained VT & $24.1 \%$ & $23.3 \%$ & NS \\
Catecholamine sensitive & $44.8 \%$ & $55.0 \%$ & NS \\
Multifocal ablation sites & $41.2 \%$ & $33.3 \%$ & NS \\
Scar zone & $21.7 \%$ & $17.2 \%$ & NS \\
Low voltage zone & $17.4 \%$ & $19.0 \%$ & NS \\
Acute success & $73.9 \%$ & $65.5 \%$ & NS \\
VT recurrence & $26.1 \%$ & $27.6 \%$ & NS \\
Repeat ablation & $13.0 \%$ & $8.6 \%$ & NS \\
\hline
\end{tabular}

$\mathrm{HR}$, heart rate; RVOT-VT, right ventricular outflow tract-ventricular tachycardia; VT, ventricular tachycardia 
RV body septum, $6.7 \%$ vs. $0.6 \%, p=0.10$ ). There was no statistical difference in the percentage of scar zone distribution between male and female (RVOT free wall, $6.7 \%$ vs. $15.9 \%, \mathrm{p}=0.18$; RVOT septum, $3.3 \%$ vs. $7.9 \%, \mathrm{p}=0.37$; RV body free wall, $13.3 \%$ vs. $23.8 \%$, $\mathrm{p}=0.24$; RV body septum, $6.7 \%$ vs. $0, \mathrm{p}=0.10$, Table 3 ).

\section{The outcome after catheter ablation}

In total, 81 patients passed catheter 3D mapping ablation ( 23 male and 58 female patients), and the mean follow up time was 26.9 months ( $26.9 \pm 32.8$ months). The acute success rate was similar in the two studied groups ( $73.9 \%$ vs. $65.5 \%, p=0.47$ ). Despite the fact that most patients had monomorphic VT, multifocal ablation sites were needed in 7 male patients and 13 female patients. We tried to analyze the sites where ablation was successful and the correlation with frequencies of scar zone or of low voltage zone and there were no differences between male and female patients. The ablation performed according to the result of pacemap locations was successful in $34.8 \%$ of male patients and $36.2 \%$ of female patients. The overall VT recurrence rate was likewise similar between the two groups $(26.1 \%$ vs. $27.6 \%, \mathrm{p}=0.89)$. Three male and five female patients had to pass catheter ablation a second time (Table 4).

\section{Discussion}

\section{Main findings}

The present study was focused on gender differences in electro-anatomic characteristics and catheter ablation in RVOT-VT, which according to the authors' best knowledge, have never been previously reported. Females were proved to have more low voltage zone in the RVOT free wall, lower mean RV voltage, and shorter QRS duration as compared with males. The acute success rate, repetitive ablation rate and VT recurrence rate did not differ between genders.

\section{Gender differences in electrophysiological characteristics}

Differences in electrophysiological characteristics between genders have been reported in several recent studies. Women have been noted to have higher heart rates at rest, longer corrected QT intervals, shorter sinus node recovery time, and longer ventricular effective refractory periods compared with men (Bernal and Moro 2006). Differences in gender hormones may explain some of these findings, but precisely how is still not well understood. For instance, variations in arrhythmia frequency with respect to the menstrual cycle have been observed. In addition, an increase in arrhythmia frequency or the new onset of arrhythmias has been noted during pregnancy (Yarnoz and Curtis 2008). Differences have also been documented in the incidence and prevalence of specific arrhythmias, including atrial fibrillation, other various supraventricular tachycardias, and sudden cardiac death. Gender differences in pulmonary vein and left atrium action potential characteristics were noted in the animal study (Tsai et al. 2011). In the human beings, female gender with atrial fibrillation could predict the presence of superior vena cava ectopic beats (Lee et al. 2005). Women have also a higher prevalence of multiple accessory pathways and orthodromic atrio-ventricular re-entrant tachycardia in the case of pre-excitation syndrome (Huang et al. 2011). In atrioventricular nodal re-entrant tachycardia, both the antegrade fast and slow pathways effective refractory periods in women were significantly shorter than those in men (Suenari et al. 2010).

Gender-specific differences exist in the incidence and age distribution of the various types of VT (Nakagawa et al. 2002). Nevertheless, limited information was noted from currently available literature. Women were found to have longer ventricular effective refractory periods in comparison to men (Liu et al. 2004). Women have also higher incidence of congenital and acquired long QT syndrome, but less ventricular tachycardia/fibrillation-related sudden cardiac death (Bernal and Moro 2006). In idiopathic ventricular tachycardia including RVOT-VT, gender-specific differences exist in the incidence and age distribution. The incidence of RVOT-VT in female is higher than that in males (Bernal and Moro 2006) but gender was not associated with the outcome after catheter ablation (Tanaka et al. 2011). Moreover, the same authors have reported that in the patients with idiopathic ventricular arrhythmias, males are prone to have tachycardia induced cardiomyopathy.

\section{Mechanisms of gender differences}

Several mechanisms have been proposed to explain the gender differences in arrhythmias, and one of those is associated with sex hormones (Chen et al. 1999). Sex hormones could regulate the expression of cardiac ion channels. Progesterone increases delayed rectifier $\mathrm{K}^{+}$ current (Iks) through the nitric oxide production pathway and prevents cyclic adenosine monophosphate enhancement of L-type $\mathrm{Ca}^{2+}$ current (Rosano et al. 1996). 
Other possible mechanisms are different distributions of ion channels between genders. James et al. (2004) reported gender-related differences in ventricular myocyte repolarization in the guinea pig. They have found in their study that IKs and inward rectifier $\mathrm{K}^{+}$ current were different between genders regardless of menstrual cycle. Gaborit et al. (2010) further reported that male and female human hearts had significant differences in ion-channel subunit composition, with female hearts showing decreased expression of a number of repolarizing ion-channels. The autonomic nervous system could also play the role. Autonomic regulation, contributing to different cardiac electrophysiology (Kapa et al. 2010, Yang et al. 2013) might explain gender differences in various arrhythmias (Dart et al. 2002, Hu et al. 2009, Morillo et al. 1994). Arg16Gly in $\beta 2-$ adrenoceptor is significantly associated with idiopathic ventricular outflow tract tachycardias in the Chinese Han population (Ran et al. 2010) which suggests the possible roles of sympathetic system in the RVOT-VT. In summary, gender differences might be attributed to multiple factors.

\section{The outcome after catheter ablation}

The gender differences in outcomes after catheter ablation for different arrhythmias have been recently reported. For instance, outcomes of catheter ablation for atrial fibrillation in women were worse than in men, probably due to later referral and older age in the women in reported study (Santangeli et al. 2011). Similar ablation results regarding differences between the genders were observed in atrioventricular nodal and atrioventricular reentrant tachycardia. With regard to idiopathic VTs, no gender-related differences in outcome of catheter ablation have been found (Tanaka et al. 2011). This study also shows that successful ablation rates, recurrence rates, and necessary repetitive operations were similar between genders. Idiopathic RVOT-VT is a relatively benign ventricular arrhythmia, and prognosis should rely on underlying conditions and comorbidities, rather than on the arrhythmia itself. Ventura et al. (2007) reported decennial follow-up in 133 patients (77 females; $39 \pm 13$ years) with RVOT-VT for $135 \pm 68$ months and $127(95 \%)$ survived, while six (5\%) died but from noncardiac diseases. In this study, ablation was performed in middle-aged groups with relatively few comorbidities and preserved left ventricular function. The modern technique of catheter ablation has a high success rate and low complications, which explains why the acute success rate and recurrence rate didn't differ between the two groups. The recent study also revealed gender difference in mutation carriers in the lamin $\mathrm{A} / \mathrm{C}$ gene (LMNA) when male had a worse prognosis due to a higher prevalence of malignant ventricular arrhythmias and end-stage heart failure (van Rijsingen et al. 2013).

\section{Limitations of the study}

In this study, some patients had RV regional dyskinesia on echocardiographic examination, while no cardiac magnetic resonance imaging (MRI) was performed to evaluate the presence of RV dysplasia. However, this is a universal limitation of registry data. According to one previous report, MRI abnormalities could still be detected in patients with idiopathic RVOTVT without other evidence of ARVC (Joshi and Wilber 2005).

\section{Conclusions}

The present study provides further evidence of the gender differences in electroanatomical mapping findings. Females had shorter QRS duration, lower right ventricular voltage, and more low voltage zone in the RVOT free wall than males. Although the possible mechanisms are not clear, our findings suggest differences in ventricular remodeling between genders in patients with idiopathic RVOT-VT. The RVOT free wall was the prominent region, which was not associated with different VT incidences. Those findings suggest that ROVT low voltage might be the remodeling result after VT, rather than the cause. The outcome after catheter ablation was similar between genders, what corresponded with the previous report (Tanaka et al. 2011). Studies on gender-specific differences in arrhythmia will lead to a better understanding of its mechanism(s) and provide valuable information for the development of optimal treatment strategies.

\section{Conflict of Interest}

There is no conflict of interest.

\section{Acknowledgements}

Supported by PRVOUK-P35/LF1/5 and SVV266503/2013. 


\section{References}

ARYA A, PIORKOWSKI C, SOMMER P, GERDS-LI JH, KOTTKAMP H, HINDRICKS G: Idiopathic outflow tract tachycardias: current perspectives. Herz 32: 218-225, 2007.

BERNAL O, MORO C: Cardiac arrhythmias in women. (in Spanish) Rev Esp Cardiol 59: 609-618, 2006.

CHEN YJ, LEE SH, HSIEH MH, HSIAO CJ, YU WC, CHIOU CW, CHEN SA: Effects of 17beta-estradiol on tachycardia-induced changes of atrial refractoriness and cisapride-induced ventricular arrhythmia. J Cardiovasc Electrophysiol 10: 587-598, 1999.

DART AM, DU XJ, KINGWELL BA: Gender, sex hormones and autonomic nervous control of the cardiovascular system. Cardiovasc Res 53: 678-687, 2002.

GABORIT N, VARRO A, LE BOUTER S, SZUTS V, ESCANDE D, NATTEL S, DEMOLOMBE S: Gender-related differences in ion-channel and transporter subunit expression in non-diseased human hearts. J Mol Cell Cardiol 49: 639-646, 2010.

HODGKINSON KA, PARFREY PS, BASSETT AS, KUPPRION C, DRENCKHAHN J, NORMAN MW, THIERFELDER L, STUCKLESS SN, DICKS EL, MCKENNA WJ, CONNORS SP: The impact of implantable cardioverter-defibrillator therapy on survival in autosomal-dominant arrhythmogenic right ventricular cardiomyopathy (ARVD5). J Am Coll Cardiol 45: 400-408, 2005.

HU YF, HUANG JL, WU TJ, HIGA S, SHIH CM, TAI CT, LIN YJ, CHANG SL, LO LW, TA-CHUAN T, CHANG CJ, TSAI WC, LEE PC, TSAO HM, ISHIGAKI S, OYAKAWA A, CHEN SA: Gender differences of electrophysiological characteristics in focal atrial tachycardia. Am J Cardiol 104: 97-100, 2009.

HUANG SY, HU YF, CHANG SL, LIN YJ, LO LW, TUAN TC, LEE PC, LI CH, SUENARI K, CHAO TF, TAI CT, CHIANG CE, CHEN SA: Gender differences of electrophysiologic characteristics in patients with accessory atrioventricular pathways. Heart Rhythm 8: 571-574, 2011.

HUMMEL JD, STRICKBERGER S, DAOUD E, NIEBAUER M, BAKR O, MAN KC, WILLIAMSON BD, MORADY F: Results and efficiency of programmed ventricular stimulation with four extrastimuli compared with one, two, and three extrastimuli. Circulation 90: 2827-2832, 1994.

JAMES AF, ARBERRY LA, HANCOX JC: Gender-related differences in ventricular myocyte repolarization in the guinea pig. Basic Res Cardiol 99: 183-192, 2004.

JOSHI S, WILBER DJ: Ablation of idiopathic right ventricular outflow tract tachycardia: current perspectives. J Cardiovasc Electrophysiol 16 (Suppl 1): S52-S58, 2005.

KAPA S, VENKATACHALAM KL, ASIRVATHAM SJ: The autonomic nervous system in cardiac electrophysiology: an elegant interaction and emerging concepts. Cardiol Rev 18: 275-284, 2010.

LEE SH, TAI CT, HSIEH MH, TSAO HM, LIN YJ, CHANG SL, HUANG JL, LEE KT, CHEN YJ, CHENG JJ, CHEN SA: Predictors of non-pulmonary vein ectopic beats initiating paroxysmal atrial fibrillation: implication for catheter ablation. $J$ Am Coll Cardiol 46: 1054-1059, 2005.

LIU XK, JAHANGIR A, TERZIC A, GERSH BJ, HAMMILL SC, SHEN WK: Age- and sex-related atrial electrophysiologic and structural changes. Am J Cardiol 94: 373-375, 2004.

LUIBA I, JONSSON A, SAFSTROM K, WALFRIDSSON H: Gender-related differences in patients with atrioventricular nodal reentry tachycardia. Am J Cardiol 97: 384-388, 2006.

MARCHLINSKI FE, DEELY MP, ZADO ES: Sex-specific triggers for right ventricular outflow tract tachycardia. Am Heart J 139: 1009-1013, 2000.

MARCUS FI, MCKENNA WJ, SHERRILL D, BASSO C, BAUCE B ET AL.: Diagnosis of arrhythmogenic right ventricular cardiomyopathy/dysplasia: proposed modification of the Task Force Criteria. Eur Heart J 31: 806$814,2010$.

MORILLO CA, KLEIN GJ, THAKUR RK, LI H, ZARDINI M, YEE R: Mechanism of 'inappropriate' sinus tachycardia. Role of sympathovagal balance. Circulation 90: 873-877, 1994.

NAKAGAWA M, TAKAHASHI N, NOBE S, ICHINOSE M, OOIE T, YUFU F, SHIGEMATSU S, HARA M, YONEMOCHI H, SAIKAWA T: Gender differences in various types of idiopathic ventricular tachycardia. J Cardiovasc Electrophysiol 13: 633-638, 2002. 
RAN YQ, LI N, YANG Y, CHEN JZ, FENG L, ZHANG S, PU JL: Beta2-adrenoceptor gene variant Arg16Gly is associated with idiopathic ventricular outflow-tract tachycardia. Chin Med J (Engl) 123: 2299-2304, 2010.

ROSANO GMC, LEONARDO F, SARREL PM, BEALE CM, DE LUCA F, COLLINS P: Cyclical variation in paroxysmal supraventricular tachycardia in women. Lancet 347: 786-788, 1996.

SANTANGELI P, DI BIASE L, PELARGONIO G, NATALE A: Outcome of invasive electrophysiological procedures and gender: are males and females the same? J Cardiovasc Electrophysiol 22: 605-612, 2011.

SOMBERG JC, PRESTON RA, RANADE V, CVETANOVIC I, MOLNAR J: Gender differences in cardiac repolarization following intravenous sotalol administration. J Cardiovasc Pharmacol Ther 17: 86-92, 2012.

SUENARI K, HU YF, TSAO HM, TAI CT, CHIANG CE, LIN YJ, CHANG SL, LO LW, TA-CHUAN T, LEE PC, TUNG NH, HUANG SY, WU TJ, CHEN SA: Gender differences in the clinical characteristics and atrioventricular nodal conduction properties in patients with atrioventricular nodal reentrant tachycardia. J Cardiovasc Electrophysiol 21: 1114-1119, 2010.

TADA H, ITO S, NAITO S, KUROSAKI K, UEDA M, SHINBO G, HOSHIZAKI H, OSHIMA S, NOGAMI A, TANIGUCHI K: Prevalence and electrocardiographic characteristics of idiopathic ventricular arrhythmia originating in the free wall of the right ventricular outflow tract. Circ J 68: 909-914, 2004.

TANAKA Y, TADA H, ITO S, NAITO S, HIGUCHI K, KUMAGAI K, HACHIYA H, HIRAO K, OSHIMA S, TANIGUCHI K, AONUMA K, ISOBE M: Gender and age differences in candidates for radiofrequency catheter ablation of idiopathic ventricular arrhythmias. Circ J 75: 1585-1591, 2011.

TSAI WC, CHEN YC, LIN YK, CHEN SA, CHEN YJ: Sex differences in the electrophysiological characteristics of pulmonary veins and left atrium and their clinical implication in atrial fibrillation. Circ Arrhythm Electrophysiol 4: 550-559, 2011.

VAN RIJSINGEN IA, NANNENBERG EA, ARBUSTINI E, ELIOTT PM, MOGENSEN J, HERMANS-VAN AST JF, VAN DER KOOI AJ, VAN TINTELEN JP, VAN DEN BERG MP, GRASSO M, SERIO A, JENKINS S, ROWLAND C, RICHARD P, WILDE AA, PERROT A, PANKUWEIT S, ZWINDERMAN AH, CHARRON P, CHRISTIAANS I, PINTO YM: Gender-specific differences in major cardiac events and mortality in lamin A/C mutation carriers. Eur J Heart Fail 15: 376-384, 2013.

VENTURA R, STEVEN D, KLEMM HU, LUTOMSKY B, MÜLLERLEILE K, ROSTOCK T, SERVATIUS H, RISIUS T, MEINERTZ T, KUCK KH, WILLEMS S: Decennial follow-up in patients with recurrent tachycardia originating from the right ventricular outflow tract: electrophysiologic characteristics and response to treatment. Eur Heart J 28: 2338-2345, 2007.

YANG SG, MLČEK M, KITTNAR O: Estrogen can modulate menopausal women's heart rate variability. Physiol Res 62 (Suppl 1): S165-S171, 2013.

YARNOZ MJ, CURTIS AB: More reasons why men and women are not the same (gender differences in electrophysiology and arrhythmias). Am J Cardiol 101: 1291-1296, 2008. 